\title{
(6) OPEN ACCESS \\ Study quality on groin injury management remains low: a systematic review on treatment of groin pain in athletes
}

\author{
Andreas Serner, ${ }^{1,2}$ Casper H van Eijck, ${ }^{3}$ Berend R Beumer, ${ }^{3}$ Per Hölmich, ${ }^{1,2}$ \\ Adam Weir, ${ }^{1}$ Robert-Jan de Vos ${ }^{4}$
}

\begin{abstract}
- Additional material is published online only. To view please visit the journal online (http://dx.doi.org/10.1136/ bjsports-2014-094256).

${ }^{1}$ Aspetar Sports Groin Pain Center, Orthopaedic and Sports Medicine Hospital, Doha, Qatar ${ }^{2}$ Arthroscopic Center Amager, SORC-C, Copenhagen University Hospital, AmagerHvidovre, Denmark

${ }^{3}$ Department of Surgery, Erasmus University Medical

Centre, Rotterdam, The Netherlands

${ }^{4}$ Department of Orthopaedics, Erasmus University Medical Centre, Rotterdam, The Netherlands
\end{abstract}

\section{Correspondence to} Dr Robert-Jan de Vos, Department of Orthopaedics, Erasmus Medical Centre, PO Box 2040, Rotterdam 3000 CA, The Netherlands; r.devos@erasmusmc.nl

Accepted 10 January 2015 Published Online First 29 January 2015

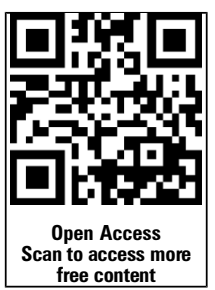

CrossMark

\section{To cite: Serner $A$,} van Eijck $\mathrm{CH}$, Beumer BR, et al. Br I Sports Med 2015;49:813

\begin{abstract}
Background Groin pain in athletes is frequent and many different treatment options have been proposed. The current level of evidence for the efficacy of these treatments is unknown.
\end{abstract}

Objective Systematically review the literature on the efficacy of treatments for groin pain in athletes.

Methods Nine medical databases were searched in May 2014. Inclusion criteria: treatment studies in athletes with groin pain; randomised controlled trials, controlled clinical trials or case series; $n>10$; outcome measures describing number of recovered athletes, patient satisfaction, pain scores or functional outcome scores. One author screened search results, and two authors independently assessed study quality. A best evidence synthesis was performed. Relationships between quality score and outcomes were evaluated. Review registration number CRD42014010262.

Results 72 studies were included for quality analysis. Four studies were high quality. There is moderate evidence that, for adductor-related groin pain, active exercises compared with passive treatments improve success, multimodal treatment with a manual therapy technique shortens the time to return to sports compared with active exercises and adductor tenotomy improves treatment success over time. There is moderate evidence that for athletes with sportsman's hernia, surgery results in better treatment success then conservative treatment. There was a moderate and inverse correlation between study quality and treatment success ( $p<0.001, r=-0.41$ ), but not between study quality and publication year $(p=0.09, r=0.20)$.

Conclusions Only $6 \%$ of publications were high quality. Low-quality studies showed significantly higher treatment success and study quality has not improved since 1985. There is moderate evidence for the efficacy of conservative treatment (active exercises and multimodal treatments) and for surgery in patients with adductor-related groin pain. There is moderate evidence for efficacy of surgical treatment in sportsman's hernia.

\section{INTRODUCTION}

Acute and long-standing groin pain are frequent problems in sports involving rapid directional changes, ${ }^{1} 2$ and frequently lead to absence from sporting activities. It is estimated that $5-18 \%$ of all sports injuries are groin-related. ${ }^{3}$

The groin region has a complex anatomy with a large number of potential pain-generating structures. Symptoms may arise from systemic, gynaecological, urogenital, gastrointestinal, neurological and musculoskeletal structures. ${ }^{4}$ This can make groin pain terminology confusing, resulting in difficulties with interpretation of research results.

The natural history of most groin injuries in sport is favourable after a short period of relative rest. ${ }^{5}$ However, some injuries can result in longer rehabilitation time and may even become longstanding. It is known that long-standing groin pain can be resistant to many treatment options and can have slow recovery times. ${ }^{6}$

Three systematic reviews have been published on the treatment of groin pain in athletes. ${ }^{4}{ }^{8}{ }^{8} \mathrm{In}$ 2008, the first review included all types of treatment, but a quality assessment was not performed for 39 out of $45(87 \%)$ studies, due to a subjective consensus on sufficient level of evidence based on individual study design. ${ }^{4}$ The authors were not able to provide clear recommendations based on the available evidence, instead they described that conservative management was usually tried initially, and surgery might be indicated following unsuccessful treatment.

Two further systematic reviews, from 2009 and 2013, only included studies on the effectiveness of conservative treatment. ${ }^{78} \mathrm{~A}$ thorough evaluation of exercise interventions, aiming to strengthen the hip and abdominal musculature, was performed in one review. ${ }^{7}$ All study designs were eligible for inclusion, but only five studies were included and assessed with a modified generic quality appraisal tool. Although the authors conclude that exercise should be a key component in the treatment of groin pain in athletes, the overall evidence base was poor. A recent Cochrane review focused on randomised controlled trials (RCTs) and quasi-RCTs only. This limited the inclusion to two studies, which were evaluated with a seven-point bias assessment. The authors concluded that the two studies provided insufficient evidence to advise a specific conservative treatment for exercise-related groin pain. ${ }^{8}$

The methodology used in the previous reviews has proven insufficient to provide a clear overview including quality considerations of all available literature on the treatment of the wide spectrum of groin pain in athletes.

We examined the currently available literature on the efficacy of conservative and surgical treatment options for groin pain in athletes. It assesses studies of all levels of evidence, with a focus on high study quality, to provide recommendations for clinical practice and guide further research. A secondary aim was to correlate the study quality with treatment success, percentage of athletes returning to play, time to return to play (RTP) and publication year. 


\section{METHODS}

Registration in the PROSPERO International prospective register of systematic reviews was performed prior to study initiation (registration number CRD42014010262).

\section{Literature search}

The databases PubMed, EMBASE, CINAHL, Medline OvidSP, Scopus, Google Scholar, Web of Science, Sportdiscus and Cochrane Library were searched without time limits in May 2014. The complete electronic search is shown in online supplementary table S1. Manual screening of the reference lists of the eligible studies was performed to include other potential eligible studies. The literature search was assisted by a biomedical information specialist (WM Bramer).

\section{Study selection}

Two independent reviewers (R-JdV and $\mathrm{BB}$ ) assessed all potentially eligible studies identified by the search strategy. The eligibility criteria were:

1. Athletes with a diagnosis of groin pain which was treated either conservatively or surgically;

2. A quantitative outcome measure in terms of treatment success, recovery rate, percentage of athletes returning to play after treatment, pain scores, functional outcome scores or patient satisfaction;

3. Study design was a RCT, prospective or retrospective controlled study, case-control study, or case series with $n>10$;

4. The article was written in English.

Studies on intra-articular hip pathologies (eg, osteoarthritis and femoroacetabular impingement) and isolated nerve injuries were excluded. All titles and/or abstracts were assessed by two independent reviewers ( $\mathrm{R}-\mathrm{JdV}$ and $\mathrm{BB}$ ) and, subsequently, relevant articles were acquired. If online access to the articles was unavailable, authors of these articles were contacted for further information. All relevant articles were read in full text by the reviewer to assess whether eligibility criteria were met.

\section{Data extraction}

One reviewer (R-JdV), blinded from the quality assessment, recorded publication data, number of participants, study design, diagnosis, intervention and, if applicable, control group(s), duration of follow-up from baseline (for primary outcome measure or, if not applicable, the last follow-up time point) and outcome, using standardised data extraction forms. Primary outcomes were extracted from the published articles to assess the treatment success of the interventions.

If the outcome was not defined as primary or secondary, the most relevant outcome was extracted. The treatment success was hierarchically defined in terms of the percentage of recovered athletes, percentage of excellent or good patient satisfaction, improvement in pain scores, improvement in functional outcome scores or percentage of athletes returning to play. Improvement in pain scores or functional scores was measured as percentage of athletes with predefined successful outcome or as a fraction of the improvement compared with the baseline measure. $^{9}$

\section{Quality assessment}

For assessment of the studies we used a modified Downs and Black (D\&B) scale (see online supplementary table S2). The D\&B scale is suitable to assess RCTs and non-randomised trials, and has shown good reliability. ${ }^{10} \mathrm{~A}$ higher score on the D\&B scale is indicative of better methodological quality. The original published tool comprises 27 items with a maximum score of 32 ; the maximum score for item 5 , regarding principal confounders, is 2 , and the last item evaluating the power of the study is scored from 0 to 5 . However, in line with previous studies, the multiple score on a single item was omitted due to its potential ambiguity. ${ }^{11} 12$ The tool in our review, therefore, consists of 27 questions with a maximum score of 27 .

We judged each study as having a high $(\geq 19 / 27)$ or low $(<19 / 27)$ quality as modified from a previous study. ${ }^{12}$ The quality assessments of the included studies were used to categorise the level of evidence. Studies with high quality (D\&B score $\geq 19 / 27$ ) were included in the final analysis for determining the efficacy of treatment in athletes with groin pain.

We also used the quality scores to evaluate the relationships with treatment success, percentage of athletes returning to play, time to RTP and publication year in all initially included studies. The Pearson's correlation coefficient analysis was used to examine the correlation between these variables if data were not skewed. The correlation coefficient $(r)$ was interpreted as no association when 0.0 , weakly positive when 0.2 , moderately positive when 0.5 , strongly positive when 0.8 and perfectly positive when $1.00 .^{13}$ Statistics were performed using SPSS V.20.0.0 (SPSS Science Inc, Chicago, Illinois, USA), and significance was considered for $\mathrm{p}$ values less than 0.05 .

The types of treatment (conservative/surgical) and injury (acute groin injury/overuse groin injury) were analysed separately. When possible, we also evaluated subgroups of patients with adductor-related, iliopsoas-related, inguinal-related and pubic-related groin pain. ${ }^{2}$

Two authors (AS and AW) independently assessed the quality of included studies using the modified D\&B forms. If there was disagreement on an item, it was discussed between the two reviewers. A consensus was reached in all cases, which precluded the need for a decisive independent third reviewer (R-JdV).

\section{Best evidence synthesis}

The heterogeneity of the data was evaluated after assessing the number of included high-quality studies. Data could be pooled if there was methodological homogeneity and $\mathrm{I}^{2}$ statistics would be performed if there was homogeneity of data. If data could not be pooled because of heterogeneity, a best evidence synthesis was carried out consisting of a qualitative analysis with five levels of evidence, whereof only the highest two levels of evidence were attainable due to the quality criteria: ${ }^{9} 14$

1. Strong evidence: provided by two or more studies with high quality and by generally consistent findings in all studies ( $\geq 75 \%$ of the studies reported consistent findings).

2. Moderate evidence: provided by one study with high quality and/or two or more studies with low quality and by generally consistent findings in all studies $(\geq 75 \%$ of the studies reported consistent findings).

3. Limited evidence: provided by only one study with low quality.

4. Conflicting evidence: inconsistent findings in multiple studies ( $<75 \%$ of the studies reported consistent findings).

5. No evidence: when no studies could be found.

\section{RESULTS}

\section{Literature search}

The initial search yielded 5380 records and, after removing duplicates, 2216 articles were screened using the title and/or abstract. Ninety-five studies were identified as potentially relevant, for which we aimed to retrieve full-text articles. Two articles could not be retrieved, even after contacting the authors, 
who did not have copies of their own publications. Citation tracking did not lead to any additional relevant articles. After reviewing the full text of 93 articles, 21 articles were excluded and 72 articles $^{6}{ }^{15-85}$ met the inclusion criteria (figure 1).

\section{Description of included studies}

Supplementary table S3 presents the characteristics of the included studies. Data extraction was performed in the 72 studies included, and a detailed description of the studies is provided regarding year of publication, study design, participants, diagnosis, intervention groups, control groups, duration of follow-up and outcomes.

Owing to heterogeneity of the established diagnoses, interventions, outcome measures, follow-up times and methodological quality, it was not possible to perform statistical pooling of the data. ${ }^{86}$

\section{Study design}

Sixty-five of the 72 studies were case series, of which 52 were retrospective and 13 prospective. Two studies were controlled clinical trials ${ }^{77} 78$ and five were RCTs. ${ }^{6} 33666976$ Two of the RCTs reported concealing their allocation and blinding of the assessors of key outcomes. ${ }^{66}$ None of the RCTs reported blinding of the patients. Publication dates ranged from 1985 to 2014.

\section{Participants}

The median number of athletes included in the studies was 41 (IQR 24-73) and 95\% were male. The mean athlete age was 27.3 years (SD 4.6, range 18-43 years). The majority of the athletes in the studies included were football players $(61 \%$ of the studies). Other sports included ice hockey (7\%), Australian rules football $(6 \%)$ and rugby $(6 \%)$. In $14 \%$ of the studies the type of sports was not reported. The level of sports was reported in $56 \%$ of the studies with a mean of $61 \%$ professional and 39\% of amateur athletes. The mean symptom duration was 10.9 months (SD 4.7, range 3-21 months).

\section{Diagnostic terminology}

Thirty-three different diagnoses were used for groin pain in athletes in the 72 included studies (see online supplementary table S3). One study included acute groin injuries and 71 were on long-standing groin pain. Diagnostic criteria were frequently not reported and, if reported, many different diagnostic criteria were used (see online supplementary table S4). It should be emphasised that diagnostic criteria were very clear in some studies, but very non-specific in most of the studies. This difference could not be addressed in the online supplementary table and the criteria presented are in some cases inferred data. Seventy-one studies evaluated the treatment effect in longstanding groin pain, and one study in acute groin injuries. The following diagnoses were most frequently used: sportsman's hernia $(31 \%)$, chronic groin pain $(10 \%)$, osteitis pubis $(10 \%)$ and adductor-related groin pain (10\%). Iliopsoas-related pain (diagnosed as 'iliopsoas syndrome' or 'iliopsoas tendinitis') was diagnosed in $3 \%$ of the studies. Multiple diagnoses were established in $14 \%$ of the studies.

\section{Interventions}

The interventions were conservative in 18 (25\%) and surgical in $54(75 \%)$ studies. The conservative treatment studies included
Figure 1 Study selection flow diagram.

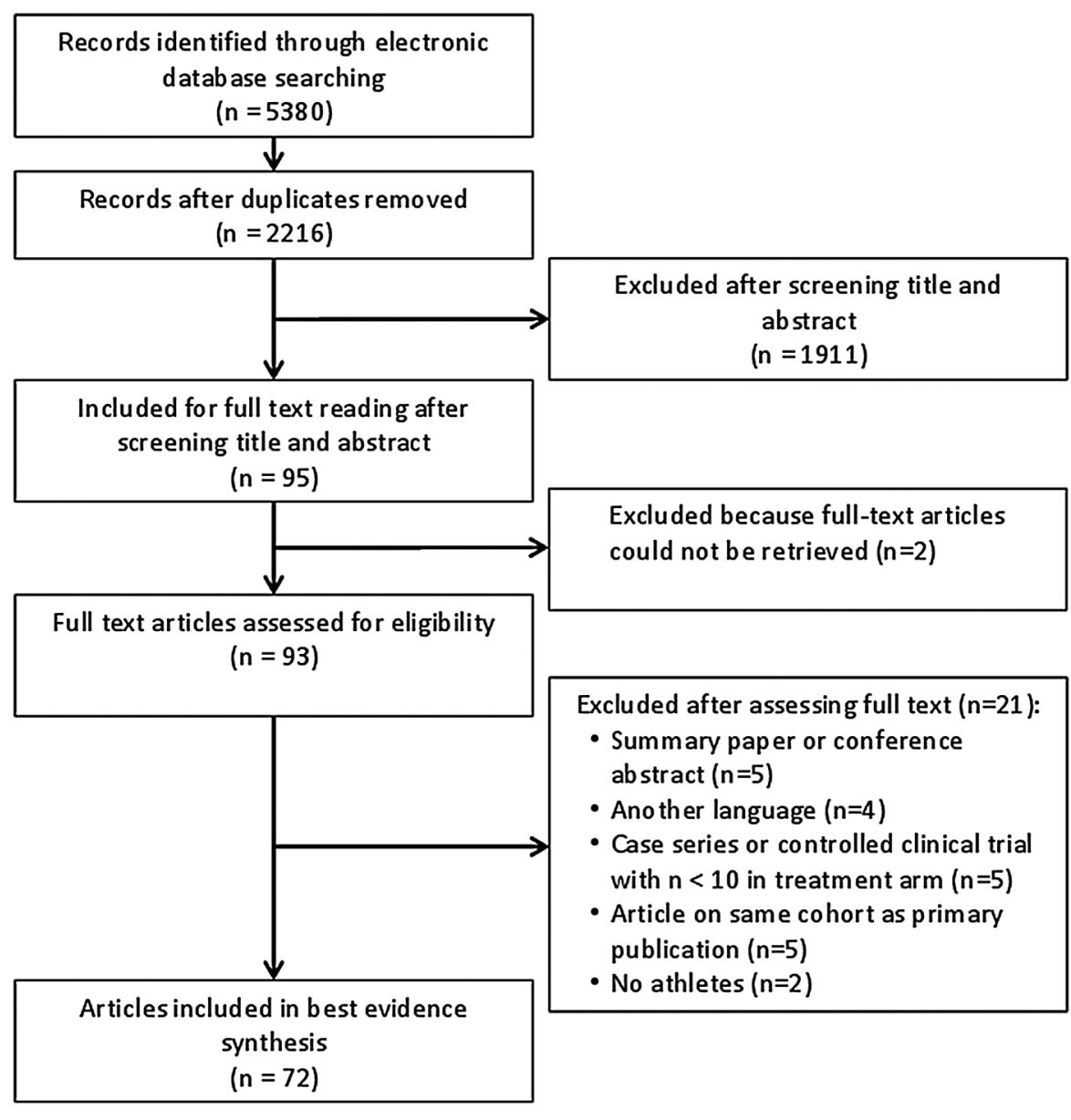


passive physical therapy modalities and/or exercise therapy (10 studies), or injection therapy (corticosteroids or dextrose, 9 studies). There were no studies focusing on the conservative treatment of sportsman's hernia with a well-described treatment protocol. The surgical studies examined open hernia repair (12 studies), laparoscopic hernia repair (10 studies) and adductor tenotomy (9 studies). Many surgical treatments were combined (16 studies), and additional neurotomy of the ilioinguinal, genitofemoral and/or iliohypogastric nerve was often performed (12 studies).

For controlled studies $(n=7)$, the control group intervention was a passive physical therapy modality or exercise therapy in three studies (43\%), local corticosteroid injection in two studies $(29 \%)$, and surgical adductor repair and wait and see in one study (14\% each).

\section{Primary outcome measures}

Many different outcome measures were used, and frequently these were not defined as being primary or secondary. Forty-three per cent of the studies described their main outcome as percentage of patients without symptoms, $21 \%$ as percentage of patients who returned to play, $17 \%$ as patient satisfaction, $14 \%$ as a pain score and $6 \%$ as a function score.

The percentage of athletes returning to play was described in $81 \%$ of the articles and the time to RTP in $38 \%$ of the articles.

\section{Outcomes}

There was a wide range in follow-up time in the 55 studies that contained these data. The mean follow-up time was 27.7 months (SD 32.1) with a range from evaluation directly after treatment to 156 months postintervention.

All studies reported a treatment success in the intervention groups (using definitions related to the percentage of recovered athletes, percentage of excellent or good patient satisfaction, improvement in pain scores, improvement in functional outcome scores, or percentage of athletes returning to play) with a mean of $84.1 \%$ of athletes (SD 16.8, range $27-100 \%$ ). A mean of $90.6 \%$ of the patients returned to play in the intervention groups (SD 11.2, range 49-100\%) and the mean reported time to RTP was 11.3 weeks (SD 8.1, range 1-38 weeks).

The mean treatment success in the control groups was $48.7 \%$ (SD 29.9, range 8-93\%). A mean of $45.8 \%$ (SD 21.9, range 14-64\%) returned to play and the mean time to RTP was 25.6 weeks (SD 5.9, range 2-17 weeks).

\section{Complications}

Forty-two articles (58\%) reported on the occurrence of complications. There were no complications in 15 of these studies (36\%) and in 27 studies (64\%) complications were mentioned. Most frequently reported were wound infections, which were reported in $13(31 \%)$ studies with a mean occurrence of 3.0\% (SD 3.5, range 1.0-14.0\%), haematomas needing evacuation (5 studies, 12\%) with a mean of $2.3 \%$ (SD 1.7, range 0.6$4.9 \%$ ), seroma formation (5 studies, $12 \%$ ) with a mean of $3.9 \%$ (SD 3.6, range 1.0-10.0\%) and neuralgia with variable durations after leaving the hospital (5 studies, 12\%) with a mean of $3.4 \%$ (SD 2.9, range 0.3-7.0\%).

\section{Quality assessment}

The quality assessment scores of all included studies are shown in table 1 . There was initial disagreement between the two reviewers in 205 of the 1944 item scores (11\%). In two of the 72 studies (3\%), this resulted in a difference between low and high quality after agreement was reached (one from high to low quality ${ }^{74}$ and one from low to high quality $\left.{ }^{81}\right)$. The percentage of agreement was lowest in items 9 (74\%), 11 (79\%), 13 (79\%) and $20(74 \%)$. The included studies scored worst on items 14 (blinding participants, 100\% absent), 15 (blinding outcome assessors, 97\% absent) and 24 (concealment treatment allocation in case of a RCT, $97 \%$ absent).

The scores ranged from 2 to 24 points with an average of 10.3 (SD 4.6). Four studies (6\%) were considered high quality (table 2). The high-quality studies were performed in patients with long-standing adductor-related groin pain $(n=3)^{6} 6681$ and sportsman's hernia with and without adductor tendinitis $(n=1){ }^{69}$ One RCT evaluated the effect of active exercise therapy in the intervention group ${ }^{6}$, one RCT evaluated the effect of multimodal treatment including a manual therapy technique $^{66}$ and one RCT assessed the effect of laparoscopic surgery. ${ }^{69}$ Two RCTs used exercise therapy as control group ${ }^{66} 69$ and one study used passive physical therapy modalities as control. $^{6}$ One prospective case series evaluated the effect of surgical adductor release. ${ }^{81}$

There was a significant moderate and inverse correlation between study quality and treatment success $(\mathrm{p}<0.001, \mathrm{r}=$ $-0.41)$. There was a weak, but non-significant correlation with quality and the percentage of athletes returning to play $(p=0.09$, $\mathrm{r}=-0.23)$ and no correlation with the time to RTP $(\mathrm{p}=0.94, \mathrm{r}=$ -0.01 ; figure 2 ). There was a non-significant weak correlation between publication year and the D\&B quality score $(\mathrm{p}=0.09$, $r=0.20$ ), so over time there was no significant improvement in the methodological quality of the studies included.

\section{Level of evidence}

The four high-quality studies showed a significant improvement after intervention at the final follow-up or predefined primary outcome time. Table 2 shows the outcomes of the high-quality studies, which all included football players.

There is moderate evidence that:

Active physical training (consisting of adductor and abdominal strengthening, and coordination exercises) is superior to passive physical therapy modalities (consisting of laser, transverse frictions, adductor stretching and electric nerve stimulation) for long-standing adductor-related groin pain. ${ }^{6}$

Multimodal treatment (consisting of adductor warming, a specific manual adductor stretch, static adductor stretches and a return to running programme) enables a quicker return to sports than active physical training (consisting of adductor and abdominal strengthening and coordination exercises, and a running programme) for long-standing adductor-related groin pain. ${ }^{66}$

Partial adductor longus release reduces pain and enables RTP over time for athletes with long-standing adductor-related groin pain. ${ }^{81}$

Laparascopic inguinal surgery (totally extraperitoneal repair) with or without surgical adductor release is more effective than conservative treatment (consisting of various types of adductor and abdominal strengthening and coordination exercises, corticosteroid injections and oral anti-inflammatory analgesics) for long-standing sportsman's hernia with or without 'adductor tendinitis'. ${ }^{69}$

There was limited evidence for all other treatment options that were evaluated in the included studies, as all low-quality studies showed an improvement in time in the intervention groups.

\section{DISCUSSION}

A total of 72 studies were suitable for inclusion in this systematic review on the treatment of groin pain in athletes. Only 


\begin{tabular}{|c|c|c|c|c|c|c|c|c|c|c|c|c|c|c|c|c|c|c|c|c|c|c|c|c|c|c|c|c|c|}
\hline Study & 1 & 2 & 3 & 4 & 5 & 6 & 7 & 8 & 9 & 10 & 11 & 12 & 13 & 14 & 15 & 16 & 17 & 18 & 19 & 20 & 21 & 22 & 23 & 24 & 25 & 26 & 27 & $\begin{array}{l}\text { Total } \\
\text { D\&B score }\end{array}$ & $\begin{array}{l}\text { High/low } \\
\text { quality }\end{array}$ \\
\hline Mozes et $a l^{15}$ & - & - & + & + & - & + & - & + & + & - & + & + & - & - & - & - & - & - & + & + & + & + & - & - & - & + & - & 12 & Low \\
\hline Smedberg et $a l^{16}$ & + & - & + & - & - & + & - & - & + & - & - & - & + & - & - & - & - & - & - & - & - & + & - & - & - & + & - & 7 & Low \\
\hline Martens et $a l^{17}$ & + & - & + & - & - & + & - & + & + & - & - & - & + & - & - & - & - & - & - & + & - & - & - & - & - & + & - & 8 & Low \\
\hline Fricker et al/ ${ }^{18}$ & + & + & + & - & - & - & - & - & - & - & - & - & + & - & - & - & - & - & - & - & - & + & - & - & - & - & - & 5 & Low \\
\hline Polglase et $a l^{19}$ & + & - & - & + & - & - & - & - & + & - & - & - & - & - & - & - & - & - & + & - & - & + & - & - & - & + & - & 6 & Low \\
\hline Shaker et $a l^{20}$ & + & - & + & - & - & + & - & - & + & - & - & - & + & - & - & - & - & - & - & + & + & + & - & - & - & + & - & 9 & Low \\
\hline Akermark and Johansson ${ }^{21}$ & + & + & + & + & - & + & - & + & - & - & - & - & - & - & - & - & - & - & + & + & - & - & - & - & - & + & - & 9 & Low \\
\hline Malycha and Lovell12 & - & + & + & + & - & + & + & - & - & + & - & - & - & - & - & + & + & + & + & + & - & + & - & - & - & + & - & 13 & Low \\
\hline Holt et $a l^{23}$ & + & - & + & + & - & + & - & - & + & - & + & + & + & - & - & - & - & - & + & + & + & + & - & - & - & - & - & 12 & Low \\
\hline Simonet et $a^{24}$ & - & - & + & + & - & - & - & - & - & - & - & - & + & - & - & - & - & - & + & - & + & - & - & - & - & - & - & 5 & Low \\
\hline Urquhart et $a l^{25}$ & + & - & - & - & - & + & - & - & - & - & - & - & - & - & - & - & - & - & + & - & + & + & - & - & - & - & - & 5 & Low \\
\hline Ingoldby ${ }^{26}$ & + & - & - & + & - & - & - & + & - & - & + & - & + & - & - & - & - & - & + & - & + & + & - & - & - & + & - & 9 & Low \\
\hline Micheli and Solomon ${ }^{27}$ & - & + & - & + & - & + & - & + & - & - & - & - & - & - & - & - & - & - & + & - & - & + & - & - & - & - & - & 6 & Low \\
\hline Evans $^{28}$ & - & - & - & + & - & - & - & - & + & - & + & - & + & - & - & - & - & - & + & - & + & + & - & - & - & + & - & 8 & Low \\
\hline Lacroix et $\left.a\right|^{29}$ & - & - & + & + & - & - & - & - & - & - & - & - & + & - & - & - & - & - & + & - & + & + & - & - & - & + & - & 7 & Low \\
\hline Holmich et $a l^{6}$ & + & + & + & + & + & + & - & - & + & + & + & + & - & - & + & + & + & + & + & + & + & + & + & + & + & + & + & 23 & High \\
\hline Brannigan et $a l^{\beta 0}$ & + & - & + & + & - & - & - & - & + & - & + & + & + & - & - & - & - & - & + & - & + & + & - & - & - & + & - & 11 & Low \\
\hline Meyers et $a^{31}$ & + & + & - & + & - & + & - & - & - & - & - & - & + & - & - & + & - & - & + & + & + & - & - & - & - & - & - & 9 & Low \\
\hline McKim and Taunton ${ }^{32}$ & + & + & + & + & - & + & + & + & - & + & - & - & + & - & - & + & + & + & + & + & + & - & + & - & - & - & - & 16 & Low \\
\hline Ekstrand and Ringborg ${ }^{33}$ & + & + & + & - & - & + & + & - & - & - & - & - & - & - & - & + & + & + & - & - & - & - & + & - & - & - & - & 9 & Low \\
\hline Irshad et $\left.a\right|^{34}$ & + & - & + & + & - & + & - & + & + & - & - & - & + & - & - & - & - & - & + & + & + & - & - & - & - & + & - & 11 & Low \\
\hline Kumar et al ${ }^{35}$ & - & + & - & + & - & + & - & - & - & + & + & - & + & - & - & - & + & + & + & + & + & + & - & - & - & - & - & 12 & Low \\
\hline $0^{\prime}$ Connell et $\left.a\right|^{36}$ & - & - & - & + & - & - & - & + & - & - & - & - & - & - & - & - & + & - & + & - & - & + & - & - & - & + & - & 6 & Low \\
\hline Srinivasan and Schuricht ${ }^{37}$ & - & + & + & + & - & + & - & + & - & - & - & - & + & - & - & - & - & - & + & - & + & + & - & - & - & - & - & 9 & Low \\
\hline Biedert et $a l^{38}$ & + & + & + & + & - & + & + & - & - & - & - & - & + & - & - & - & - & - & + & + & - & + & - & - & - & + & - & 11 & Low \\
\hline Van Der Donckt et al ${ }^{39}$ & + & - & + & + & + & + & - & - & - & - & - & - & - & - & - & + & - & - & + & - & - & - & - & - & - & - & - & 7 & Low \\
\hline Genitsaris et $a l^{40}$ & + & - & + & + & - & - & - & + & - & - & - & - & + & - & - & - & - & - & + & - & + & + & - & - & - & - & - & 8 & Low \\
\hline Kluin et $a l^{41}$ & + & - & + & + & - & + & - & - & - & - & + & - & + & - & - & + & + & - & + & - & + & + & - & - & - & + & - & 12 & Low \\
\hline Paajanen et $a l^{42}$ & - & + & + & + & - & - & - & + & - & - & + & - & + & - & - & - & - & - & + & - & + & + & - & - & - & + & - & 10 & Low \\
\hline Steele et $a l^{43}$ & + & - & - & + & - & - & - & - & + & - & - & - & - & - & - & - & - & - & + & - & - & + & - & - & - & + & - & 6 & Low \\
\hline Susmallian et al ${ }^{44}$ & + & - & - & + & - & - & - & - & - & - & - & - & - & - & - & - & - & - & + & - & + & + & - & - & - & - & - & 5 & Low \\
\hline Ahumada et $a l^{45}$ & + & - & + & + & - & - & - & - & - & - & - & - & + & - & - & - & - & - & + & - & - & + & - & - & - & - & - & 6 & Low \\
\hline Diaco et $a l^{25}$ & - & + & - & - & - & + & - & - & - & - & - & - & + & - & - & - & - & - & + & + & - & + & - & - & - & - & - & 6 & Low \\
\hline Topol et a $\left.\right|^{47}$ & + & + & + & + & - & + & + & - & + & + & + & + & + & - & - & - & - & + & + & + & + & - & - & - & - & + & - & 16 & Low \\
\hline Edelman and Selesnick ${ }^{49}$ & + & - & - & + & - & - & - & - & + & - & - & - & + & - & - & - & + & - & + & - & - & + & - & - & - & + & - & 8 & Low \\
\hline Canonico et $\left.a\right|^{48}$ & + & + & + & + & - & + & - & + & + & - & - & - & + & - & - & + & + & - & + & + & - & + & - & - & - & + & - & 14 & Low \\
\hline Schilders et al ${ }^{50}$ & + & - & + & + & - & + & - & + & + & - & + & - & + & - & - & + & + & - & + & + & - & + & - & - & - & + & - & 14 & Low \\
\hline Van Veen et $a l^{51}$ & + & - & + & + & - & + & - & - & - & - & + & - & + & - & - & + & + & - & + & + & + & + & - & - & - & - & - & 12 & Low \\
\hline Verrall et $a l^{52}$ & + & + & + & + & - & + & - & - & + & + & - & - & - & - & - & + & + & + & - & + & - & + & - & - & - & + & - & 13 & Low \\
\hline Brown et $a f^{33}$ & - & - & + & + & - & - & - & + & - & - & - & - & - & - & - & - & - & - & + & - & - & - & - & - & - & - & - & 4 & Low \\
\hline Lloyd et $\left.a\right|^{54}$ & + & + & + & + & - & + & - & + & - & - & - & - & + & - & - & - & - & - & + & - & + & + & - & - & - & - & - & 10 & Low \\
\hline
\end{tabular}


Table 1 Continued

\begin{tabular}{|c|c|c|c|c|c|c|c|c|c|c|c|c|c|c|c|c|c|c|c|c|c|c|c|c|c|c|c|c|c|}
\hline Study & 1 & 2 & 3 & 4 & 5 & 6 & 7 & 8 & 9 & 10 & 11 & 12 & 13 & 14 & 15 & 16 & 17 & 18 & 19 & 20 & 21 & 22 & 23 & 24 & 25 & 26 & 27 & $\begin{array}{l}\text { Total } \\
\text { D\&B score }\end{array}$ & $\begin{array}{l}\text { High/low } \\
\text { quality }\end{array}$ \\
\hline Meyers et $\left.a\right|^{55}$ & - & - & - & - & - & - & - & + & - & - & - & - & - & - & - & - & - & - & + & - & - & - & - & - & - & - & - & 2 & Low \\
\hline Radic and Annear ${ }^{56}$ & + & + & + & + & - & + & - & - & - & - & - & - & + & - & - & - & - & - & + & + & + & + & - & - & - & - & - & 10 & Low \\
\hline Topol and Reeves ${ }^{57}$ & + & - & - & + & - & + & + & + & + & + & - & - & - & - & - & - & - & + & + & + & - & - & - & - & - & + & - & 11 & Low \\
\hline Ziprin et $\left.a\right|^{58}$ & - & + & + & + & - & + & - & - & - & - & - & - & - & - & - & - & - & - & + & + & - & - & - & - & - & + & - & 7 & Low \\
\hline Atkinson et $a^{a^{59}}$ & - & + & - & + & - & + & + & + & + & + & - & - & - & - & - & - & - & + & + & + & + & + & - & - & - & + & - & 13 & Low \\
\hline Jansen et $a^{60}$ & + & + & + & + & - & + & - & - & + & - & + & - & + & - & - & + & + & + & - & - & + & - & - & - & - & + & + & 14 & Low \\
\hline Mann et $a l^{61}$ & + & + & + & + & + & + & - & + & - & + & + & - & + & - & - & + & - & + & + & + & + & + & - & - & - & - & - & 16 & Low \\
\hline Schilders et $a^{62}$ & + & - & - & + & + & + & - & - & + & - & - & - & + & - & - & + & + & - & + & + & + & + & - & - & + & + & - & 14 & Low \\
\hline Schlegel et $a^{63}$ & + & - & + & - & + & + & + & + & - & + & - & - & - & - & - & - & - & - & - & + & - & - & - & - & + & + & - & 10 & Low \\
\hline Weir et al ${ }^{64}$ & + & + & + & + & + & + & + & + & + & - & + & - & - & - & - & - & - & + & + & + & + & + & - & - & - & + & - & 16 & Low \\
\hline Muschaweck and Berger ${ }^{67}$ & + & + & + & + & - & + & + & - & + & + & - & - & + & - & - & + & + & + & + & + & - & + & - & - & - & + & - & 16 & Low \\
\hline Weir et al ${ }^{64}$ & + & - & + & + & + & + & + & - & + & + & - & - & + & - & - & - & - & - & - & + & + & - & - & - & + & + & - & 13 & Low \\
\hline Chernyavsky et $a^{68}$ & - & - & - & + & - & - & - & - & - & - & - & - & + & - & - & - & - & - & + & - & + & - & - & - & - & - & - & 4 & Low \\
\hline Paajanen et $a l^{42}$ & + & + & + & + & + & + & + & + & + & + & + & - & + & - & - & + & + & + & - & + & - & + & + & - & + & + & + & 21 & High \\
\hline Preskitt ${ }^{70}$ & - & - & - & + & - & - & - & - & - & - & - & - & + & - & - & - & - & - & + & - & + & - & - & - & - & - & - & 4 & Low \\
\hline Robertson et $a l^{71}$ & + & + & - & + & - & + & - & + & + & - & - & - & - & - & - & - & - & - & + & + & + & + & - & - & - & + & - & 11 & Low \\
\hline Weir et $a l^{64}$ & + & + & + & + & + & + & + & + & + & + & + & + & - & - & + & + & + & + & + & + & + & - & + & + & + & + & + & 24 & High \\
\hline Dojcinovic et $a l^{72}$ & - & - & + & + & - & + & - & - & - & - & - & - & - & - & - & + & - & - & + & - & - & + & - & - & - & + & - & 7 & Low \\
\hline Jans et $a l^{73}$ & + & - & - & + & + & + & - & + & - & - & - & - & + & - & - & - & - & - & + & - & + & + & - & - & - & - & - & 9 & Low \\
\hline Maffulli et $a l^{74}$ & + & + & + & + & + & + & + & + & + & + & - & - & - & - & - & + & - & + & + & + & - & + & - & - & + & + & - & 17 & Low \\
\hline Messaoudi et al ${ }^{75}$ & + & - & - & + & - & + & - & - & - & - & - & - & + & - & - & - & - & - & + & - & + & + & - & - & - & - & - & 7 & Low \\
\hline Comin et $a l^{76}$ & + & + & + & + & - & + & + & + & + & + & + & + & - & - & - & + & + & + & + & - & - & + & + & - & - & + & - & 18 & Low \\
\hline Economopoulos et al ${ }^{77}$ & + & + & + & + & + & + & - & + & + & + & - & - & + & - & - & - & - & + & + & - & + & - & - & - & - & + & - & 14 & Low \\
\hline Garvey and Hazard ${ }^{78}$ & - & - & - & - & - & - & - & + & - & - & - & - & - & - & - & - & - & - & - & - & - & + & - & - & - & - & - & 2 & Low \\
\hline Jakoi et al9 & + & + & - & - & - & + & - & - & - & + & - & - & - & - & - & - & + & + & + & - & - & + & - & - & - & - & - & 8 & Low \\
\hline Mei-Dan et $a^{80}$ & + & + & - & + & - & + & + & + & + & + & - & - & - & - & - & - & - & + & + & - & - & + & - & - & - & + & - & 12 & Low \\
\hline Schilders et $a^{81}$ & + & + & - & + & + & + & + & + & + & + & - & + & + & - & - & + & - & + & + & + & + & + & - & - & + & + & - & 19 & High \\
\hline Bernhardt et $a l^{82}$ & + & + & + & + & - & + & + & - & - & - & - & - & + & - & - & - & - & - & + & - & - & + & - & - & - & - & - & 9 & Low \\
\hline Cavalli et $a l^{83}$ & + & - & - & + & - & - & - & - & - & - & - & - & + & - & - & - & - & - & + & - & + & + & - & - & - & - & - & 6 & Low \\
\hline de Queiroz et $a l^{84}$ & + & + & + & + & - & - & - & + & - & - & - & - & + & - & - & - & + & - & + & - & - & + & - & - & - & - & - & 9 & Low \\
\hline Sansone et $a^{85}$ & + & + & + & + & - & + & + & - & - & + & - & - & + & - & - & - & - & + & + & + & - & - & - & - & - & - & - & 11 & Low \\
\hline Percentage of agreement/item & 94 & 81 & 82 & 97 & 86 & 81 & 94 & 94 & 74 & 96 & 79 & 82 & 79 & 100 & 100 & 92 & 93 & 85 & 96 & 74 & 85 & 94 & 99 & 99 & 99 & 82 & 100 & & \\
\hline
\end{tabular}

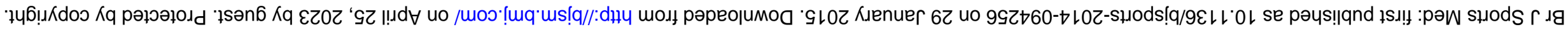




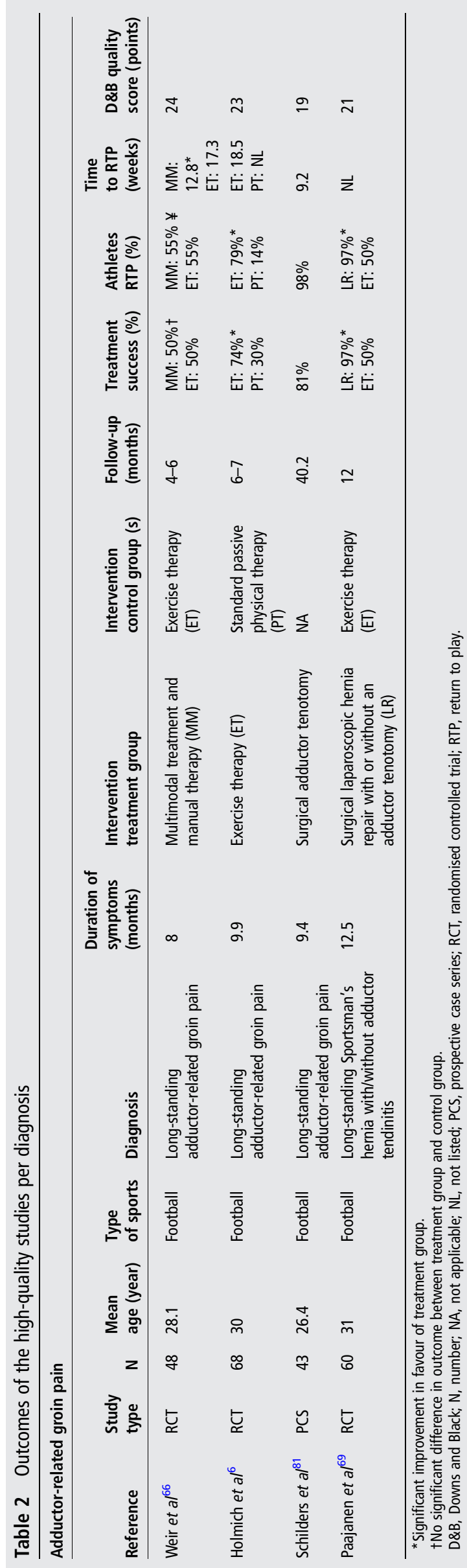

$4(6 \%)$ studies were high quality. Three of these studies were on athletes with long-standing adductor-related groin pain. According to the best evidence synthesis of this systematic review, there is currently moderate evidence that: (1) active exercise therapy improves treatment success when compared with passive treatments, (2) multimodal treatment with a manual therapy technique shortens the time to return to sports when compared to active exercise therapy and (3) adductor tenotomy improves treatment success over time.

For athletes suffering from sportsman's hernia with or without 'adductor tendinitis', there is moderate evidence that surgery results in better treatment success compared with conservative therapy. There was limited evidence for all other treatment options that were evaluated in the included studies.

An important secondary finding in this systematic review is the inverse relationship between study quality and treatment success; the higher the study quality, the lower the treatment success. Over time, the quality of studies has not improved significantly. Notably, only one study included acute groin injuries. As this study was not considered high quality, no definite conclusions can be made regarding the treatment of this type of injury. ${ }^{63}$

\section{Low-quality studies result in better outcome}

This is the first review on groin pain in athletes that demonstrated a relationship between study quality and treatment success-lower quality studies showed significantly higher treatment success. This is an important finding and highlights the fact that caution is needed when drawing conclusions based on low-quality studies. Previous studies on the management of tendinopathy also showed this inverse relationship, which is known as the 'Coleman effect'. ${ }^{87} 88$ No significant relationship was found between either the percentage of athletes returning to play or the time to RTP, and study quality. This is probably due to the fact that fewer studies could be included in these analyses, as these data were not reported in all studies, and that this type of data is more objective than treatment success, which is often defined according to a threshold set by the individual authors.

\section{Quality assessment}

We used the modified D\&B quality assessment tool to evaluate the study quality, as it is a suitable tool for controlled trials as well as for case series ${ }^{10}$ and has good reliability. ${ }^{10}$ After modification of the original tool, there were still 27 items to assess, making it a complete assessment tool. The cut-off of 19 points, to discriminate high-quality and low-quality studies, is a dichotomous approach, but was deemed necessary to perform a reasonable best evidence synthesis. The cut-off used was modified from the existing literature. ${ }^{12}$ We did not perform a separate 'risk of bias' assessment as the D\&B quality assessment tool examines the major sources of bias in its items. This is, in our opinion, a large improvement of the previous quality assessments in reviews on this topic, which have omitted a large number of studies from the quality assessment and have only used a subjectively determined evidence level cut-off, a modified generic quality appraisal tool or a seven-point bias-risk assessment. ${ }^{48}$

The lack of high-quality studies in the field is highlighted in this systematic review. Only 6\% were assessed as being high quality. The most frequent methodological flaws were the lack of a suitable control group, randomisation (including allocation concealment), and blinding of participants and those involved in 

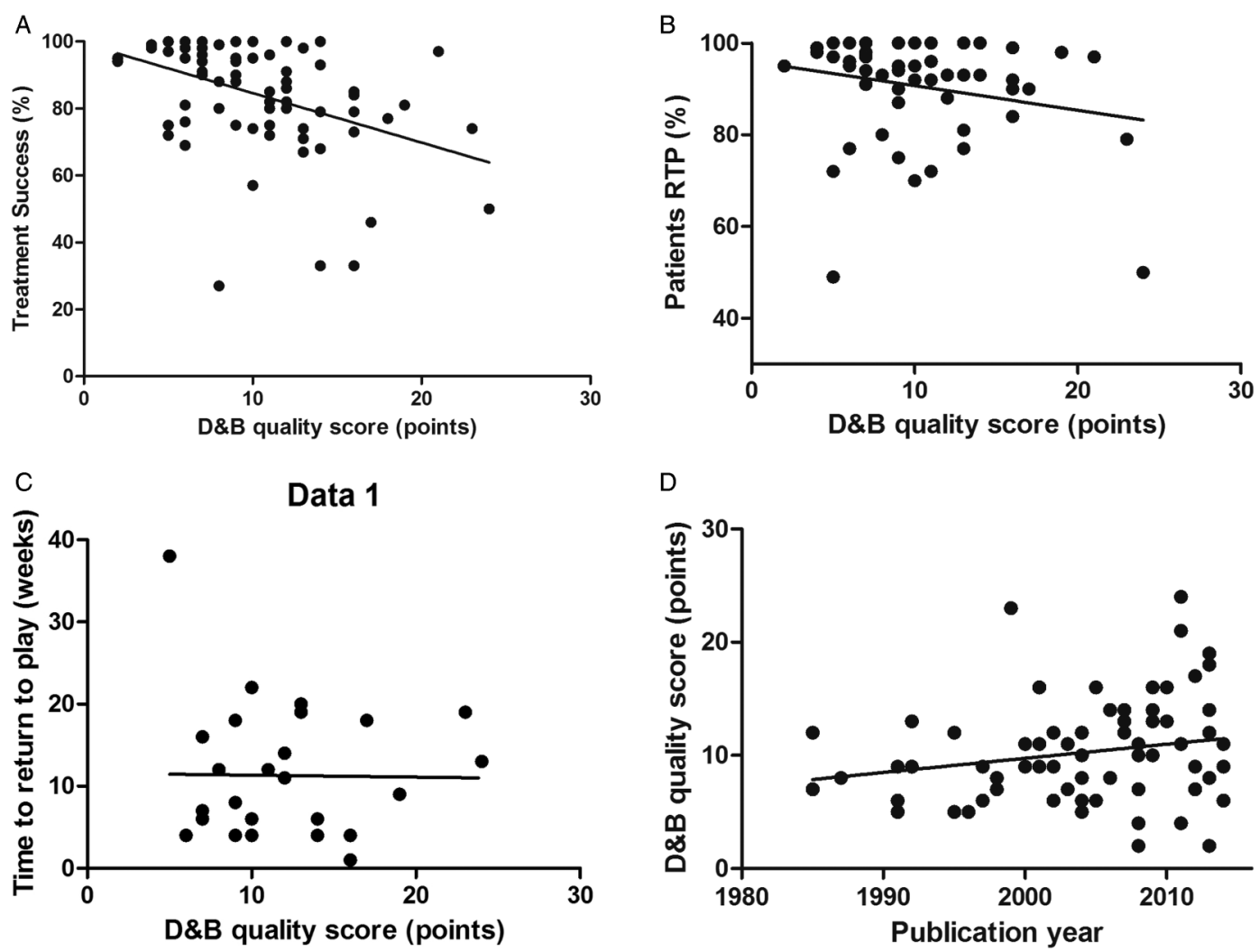

Figure 2 Correlation between quality scores and outcome or publication year. (A) Significant association between treatment success and study quality score $(p<0.001, r=-0.41)$. (B) Association between percentage of patients returning to play and study quality score $(p=0.09, r=-0.23)$. (C) Association between time to RTP in weeks and study quality score ( $p=0.94, r=-0.01)$. (D) Association between study quality score and publication year $(p=0.09, r=0.20)$. $D \& B$, Downs and Black; RTP, return to play.

the treatment. Future studies should, therefore, use these features in the study design. There has been no significant improvement in the studies from 1985 to 2014. Even in recent publications many authors fail to report on basic information, and could have benefitted from following reporting guidelines such as STROBE and CONSORT. ${ }^{89} 90$ This demonstrates that the current review process is not succeeding in enforcing these guidelines in new publications.

\section{Many different diagnoses for groin pain}

A major problem in the field of groin pain in athletes is the lack of consensus on diagnostic criteria. ${ }^{91}$ Our systematic review revealed that 33 different diagnoses were used for groin pain in athletes (see online supplementary table S3). Clear diagnostic criteria were frequently not reported and, if they were, many different diagnostic criteria were used (see online supplementary table S4). Consensus on diagnostic criteria in the field of groin pain in athletes would help to decrease heterogeneity between studies, and it would aid in interpreting and comparing studies for clinical decision-making. In a recent position statement, an expert group aimed to improve terminology for groin injuries in the inguinal region by adding a new term, 'inguinal disruption'. ${ }^{92}$ This was defined but only covers one location of groin pain in athletes. While this systematic review is not designed to propose diagnostic criteria, we would like to emphasise the need for this.

\section{Potential limitations of this systematic review}

There are a few potential limitations of this systematic review. One limitation is that we analysed the results of the predefined outcome measures, or the final follow-up measurements if there was no predefined primary outcome, which was frequently the case. This may have resulted in a bias towards shorter follow-up times, and this fact is not obvious in some cases after the data extraction. For this reason we also extracted data of the time to RTP, enabling readers to estimate the time of recovery. While only $38 \%$ of the included articles reported on the time to RTP, the mean was 11 weeks across these studies. This could be helpful for clinicians when discussing prognosis with athletes in general. The large SD of 8 weeks should also be considered in this regard.

A second limitation may be the relatively high number of disagreements between the authors in the quality assessment. There was initial disagreement in $11 \%$ of the item scores, and in 3\% this resulted in a difference between low and high quality after reaching agreement. There are two possible reasons for this result. First, a few items on the D\&B assessment form are not optimally described, especially for case series. The questions on describing the patients lost to follow-up, those on external validity and the main outcome measures had the lowest level of agreement. Interpretation of the items is difficult in some cases. For example, we chose to award a point if estimates of the random variability in the data (item 7 of the D\&B tool) were displayed for the extracted outcome measures. As we extracted multiple outcome measures, it was complex to evaluate this item in some cases. Second, the writing quality of the eligible articles was disappointing, and many authors did not follow the CONSORT or STROBE guidelines for their methods and results. The level of description in the articles makes it consequently challenging to find and interpret the requested 
information. Failure to report according to the existing guidelines will probably result in more disagreements, as reviewers are forced to rely on assumptions. A clear description of the methods and results containing all relevant information makes assessing the quality much easier.

A third potential limitation of this systematic review is the fact that we were not able to pool data for a quantitative analysis. As stated in the methods, we would only do this if there was homogeneity of data. Owing to the obvious heterogeneity of the diagnoses, interventions, outcome measures, follow-up times and methodological quality, we refrained from statistical pooling of the data. A quantitative analysis has been performed using the calculated percentages of improvement in pain and/or function scores, which has also been carried out in previous systematic reviews. ${ }^{9} 88$ As this percentage improvement is dependent on the baseline score, it is less optimal. However, it was the best available measure we could apply to enable us to explore correlations between study quality and treatment success.

A fourth limitation is the fact that we excluded all low-quality studies before performing the best evidence synthesis. Another approach could be that we only included all RCTs in the best evidence synthesis, which is a common method. ${ }^{9}$ However, we wanted to stress the importance of the quality assessment. We are aware that even multiple low-quality studies may provide useful information, but on the other hand, this systematic review highlights the high risk of bias when evaluating treatment success with methodologically flawed studies. We feel this inverse relationship between methodological quality and reported success validates our choice to not pool many lowquality studies into the evidence synthesis. Our methods were predefined and documented in the PROSPERO International prospective register of systematic reviews (registration number CRD42014010262).

\section{Recommendations for future studies}

Future studies in this field should use appropriate control groups with blinding of patients and treatment assessors, if possible. Authors should follow the CONSORT or STROBE guidelines when reporting their studies to allow better evaluation of the quality. Although only RCTs will have the possibility for the optimal quality score, this study shows that the outcome in case series can also be relevant if performed and reported well. There is also a need for high-quality studies on acute groin injuries.

\section{CONCLUSION}

There are many publications on the effect of treatments in athletes with long-standing groin pain, but very limited information on acute groin injuries. Only $6 \%$ of the included studies were high quality. These studies include different treatments, so there is no strong evidence to support any single treatment option. There is currently moderate evidence for surgical and conservative treatment of athletes with longstanding adductor-related groin pain, and for surgical treatment of athletes with sportsman's hernia. There was limited evidence for all other treatment options that were evaluated in the included studies.

Lower quality studies reported significantly higher treatment success, and study quality has not improved significantly over the past 30 years. There is a clear need for well-designed studies in this field with adequate reporting following the appropriate guidelines.

\section{What is already known?}

- Groin pain in athletes is difficult to treat and can result in prolonged absence from sporting activities.

- The best available evidence from two previous systematic reviews on treatment of groin pain in athletes was exercise therapy, but the evidence could be regarded as limited.

- A recent Cochrane review only included randomised controlled trials and concluded that there is insufficient evidence to advise a specific conservative treatment for exercise-related groin pain.

\section{What are the new findings?}

- Only $6 \%$ of the studies on treatment of athletes with groin pain are of high quality.

- There was a significant correlation between lower study quality and higher treatment success.

- For athletes with long-standing adductor-related groin pain there is moderate evidence that: (1) active exercises improve treatment success compared with passive treatments, (2) multimodal treatment with a manual therapy technique shortens the time to return to sports compared with active exercises and (3) adductor tenotomy improves treatment success over time.

- For athletes suffering from sportsman's hernia with/without adductor tendinitis, there is moderate evidence that surgery results in better treatment success compared with conservative therapy.

Acknowledgements The authors would like to thank WM Bramer (biomedical information specialist in the Erasmus University medical centre, Rotterdam, the Netherlands) for assistance in the search strategy.

Contributors R-JdV performed the search strategy, data extraction and summary, analysed and interpreted the data, and wrote the first draft of the paper. BB performed the search strategy. AS and AW performed quality assessment, interpreted the data and revised the paper. $\mathrm{CHvE}$ and $\mathrm{PH}$ interpreted the data and revised the paper. All authors gave final approval for the version to be published.

\section{Competing interests None.}

\section{Patient consent Obtained.}

Provenance and peer review Not commissioned; externally peer reviewed.

Open Access This is an Open Access article distributed in accordance with the Creative Commons Attribution Non Commercial (CC BY-NC 4.0) license, which permits others to distribute, remix, adapt, build upon this work non-commercially, and license their derivative works on different terms, provided the original work is properly cited and the use is non-commercial. See: http://creativecommons.org/ licenses/by-nc/4.0/

\section{REFERENCES}

1 Bradshaw C, McCrory P, Bell S, et al. Obturator nerve entrapment. A cause of groin pain in athletes. Am J Sports Med 1997;25:402-8.

2 Brukner P, Khan K, Brukner P. Brukner \& Khan's clinical sports medicine. 4th edn. Sydney; New York: McGraw-Hill, 2012.

3 Morelli V, Smith V. Groin injuries in athletes. Am Fam Phys 2001;64:1405-14.

4 Jansen JA, Mens JM, Backx FJ, et al. Treatment of longstanding groin pain in athletes: a systematic review. Scand J Med Sci Sports 2008;18:263-74.

5 Arnason A, Sigurdsson SB, Gudmundsson A, et al. Risk factors for injuries in football. Am J Sports Med 2004;32(Suppl. 1):5S-16S.

6 Holmich $\mathrm{P}$, Uhrskou P, Ulnits $\mathrm{L}$, et al. Effectiveness of active physical training as treatment for long-standing adductor-related groin pain in athletes: randomised trial. Lancet 1999;353:439-43. 
7 Machotka Z, Kumar S, Perraton LG. A systematic review of the literature on the effectiveness of exercise therapy for groin pain in athletes. Sports Med Arthrosc Rehabil Ther Technol 2009;1:5.

8 Almeida MO, Silva BN, Andriolo RB, et al. Conservative interventions for treating exercise-related musculotendinous, ligamentous and osseous groin pain. Cochrane Database Syst Rev 2013;6:CD009565.

9 de Vos RJ, Windt J, Weir A. Strong evidence against platelet-rich plasma injections for chronic lateral epicondylar tendinopathy: a systematic review. Br J Sports Med 2014;48:952-6.

10 Downs SH, Black N. The feasibility of creating a checklist for the assessment of the methodological quality both of randomised and non-randomised studies of health care interventions. J Epidemiol Community Health 1998:52:377-84.

11 Langham S, Langham J, Goertz HP, et al. Large-scale, prospective, observational studies in patients with psoriasis and psoriatic arthritis: a systematic and critical review. BMC Med Res Methodol 2011;11:32.

12 Samoocha D, Bruinvels DJ, Elbers NA, et al. Effectiveness of web-based interventions on patient empowerment: a systematic review and meta-analysis. J Med Internet Res 2010;12:e23.

13 Zou KH, Tuncali K, Silverman SG. Correlation and simple linear regression. Radiology 2003;227:617-22.

14 van Tulder M, Furlan A, Bombardier C, et al. Updated method guidelines for systematic reviews in the cochrane collaboration back review group. Spine (Phila Pa 1976) 2003;28:1290-9.

15 Mozes M, Papa MZ, Zweig A, et al. Iliopsoas injury in soccer players. Br J Sports Med 1985;19:168-70.

16 Smedberg SG, Broome AE, Gullmo A, et al. Herniography in athletes with groin pain. Am J Surg 1985;149:378-82.

17 Martens MA, Hansen L, Mulier JC. Adductor tendinitis and musculus rectus abdominis tendopathy. Am J Sports Med 1987;15:353-6.

18 Fricker PA, Taunton JE, Ammann W. Osteitis pubis in athletes. Infection, inflammation or injury? Sports Med 1991;12:266-79.

19 Polglase AL, Frydman GM, Farmer KC. Inguinal surgery for debilitating chronic groin pain in athletes. Med J Aust 1991;155:674-7.

20 Shaker AM, El-Kader Shaheen MA, O'Neel PJ, et al. Traumatic aseptic osteitis pubis. Ann Saudi Med 1991;11:205-8.

21 Akermark $C$, Johansson C. Tenotomy of the adductor longus tendon in the treatment of chronic groin pain in athletes. Am J Sports Med 1992;20:640-3.

22 Malycha $P$, Lovell $G$. Inguinal surgery in athletes with chronic groin pain: the 'sportsman's' hernia. Aust N Z J Surg 1992;62:123-5.

23 Holt MA, Keene JS, Graf BK, et al. Treatment of osteitis pubis in athletes: results of corticosteroid injections. Am J Sports Med 1995;23:601-6.

24 Simonet WT, Saylor IHL, Sim L. Abdominal wall muscle tears in hockey players. Int J Sports Med 1995;16:126-8.

25 Urquhart DS, Packer GJ, McLatchie GR. Return to sport and patient satisfaction levels after surgical treatment for groin disruption. Sports Exerc Inj 1996;2:37-42.

26 Ingoldby $\mathrm{CJH}$. Laparoscopic and conventional repair of groin disruption in sportsmen. Br J Surg 1997;84:213-15.

27 Micheli LJ, Solomon R. Treatment of recalcitrant iliopsoas tendinitis in athletes and dancers with corticosteroid injection under fluoroscopy. J Dance Med Sci 1997:1:7-11.

28 Evans DS. Sports hernia: the diagnosis and laparoscopic management. Sports Exerc Inj 1998;4:28-31.

29 Lacroix VJ, Kinnear DG, Mulder DS, et al. Lower abdominal pain syndrome in National Hockey League players: a report of 11 cases. Clin J Sport Med 1998;8:5-9.

30 Brannigan AE, Kerin MJ, McEntee GP. Gilmore's groin repair in athletes. J Orthop Sports Phys Ther 2000;30:329-32.

31 Meyers WC, Foley DP, Garrett WE, et al. Management of severe lower abdominal or inguinal pain in high-performance athletes. Am J Sports Med 2000;28:2-8.

32 McKim KR, Taunton JE. The effectiveness of compression shorts in the treatment of athletes with osteitis pubis. N Z J Sports Med 2001;29:70-3.

33 Ekstrand J, Ringborg S. Surgery versus conservative treatment in soccer players with chronic groin pain: a prospective randomised study in soccer players. Eur J Sports Traumatol Relat Res 2001;23:141-5.

34 Irshad K, Feldman LS, Lavoie C, et al. Operative management of "hockey groin syndrome": 12 years of experience in National Hockey League players. Surgery 2001;130:759-66.

35 Kumar S, Wilson RG, Nixon SJ, et al. Chronic pain after laparoscopic and open mesh repair of groin hernia. Br J Surg 2002;89:1476-9.

36 O'Connell MJ, Powell T, McCaffrey NM, et al. Symphyseal cleft injection in the diagnosis and treatment of osteitis pubis in athletes. Am J Roentgenol 2002;179:955-9.

37 Srinivasan A, Schuricht A. Long-term follow-up of laparoscopic preperitoneal hernia repair in professional athletes. J Laparoendosc Adv Surg Techn A 2002;12:101-6.

38 Biedert RM, Warnke K, Meyer S. Symphysis syndrome in athletes: surgical treatment for chronic lower abdominal, groin, and adductor pain in athletes. Clin I Sport Med 2003; 13:278-84.
39 Van Der Donckt K, Steenbrugge F, Van Den Abbeele K, et al. Bassini's hernial repair and adductor longus tenotomy in the treatment of chronic groin pain in athletes. Acta Orthop Belg 2003;69:35-41.

40 Genitsaris M, Goulimaris I, Sikas N. Laparoscopic repair of groin pain in athletes. Am J Sports Med 2004;32:1238-42.

41 Kluin J, Den Hoed PT, Van Linschoten R, et al. Endoscopic evaluation and treatment of groin pain in the athlete. Am J Sports Med 2004;32:944-9.

42 Paajanen $\mathrm{H}$, Syvahuoko I, Airo I. Totally extraperitoneal endoscopic (TEP) treatment of sportsman's hernia. Surg Laparosc Endosc Percutan Tech 2004;14:215-18.

43 Steele $\mathrm{P}$, Annear $\mathrm{P}$, Grove JR. Surgery for posterior inguinal wall deficiency in athletes. J Sci Med Sport 2004;7:415-21.

44 Susmallian S, Ezri T, Elis M, et al. Laparoscopic repair of 'sportsman's hernia' in soccer players as treatment of chronic inguinal pain. Med Sci Monit 2004;10: CR52-CR4.

45 Ahumada LA, Ashruf S, Espinosa-De-Los-Monteros A, et al. Athletic pubalgia: definition and surgical treatment. Ann Plast Surg 2005;55:393-6.

46 Diaco JF, Diaco DS, Lockhart L. Sports hernia. Oper Tech Sports Med 2005; 13:68-70

47 Topol GA, Reeves KD, Hassanein KM. Efficacy of dextrose prolotherapy in elite male kicking-sport athletes with chronic groin pain. Arch Phys Med Rehabil 2005;86:697-702.

48 Canonico S, Benevento R, Della Corte A, et al. Sutureless tension-free hernia repair with human fibrin glue (Tissucol) in soccer players with chronic inguinal pain: initial experience. Int J Sports Med 2007;28:873-6.

49 Edelman DS, Selesnick H. "Sports" hernia: treatment with biologic mesh (Surgisis): a preliminary study. Surg Endosc Interv Tech 2006;20:971-3.

50 Schilders E, Bismil Q, Robinson P, et al. Adductor-related groin pain in competitive athletes: role of adductor enthesis, magnetic resonance imaging, and entheseal pubic cleft injections. J Bone Joint Surg Am 2007;89:2173-8.

51 Van Veen RN, De Baat P, Heijboer MP, et al. Successful endoscopic treatment of chronic groin pain in athletes. Surg Endosc Interv Tech 2007;21:189-93.

52 Verrall GM, Slavotinek JP, Fon GT, et al. Outcome of conservative management of athletic chronic groin injury diagnosed as pubic bone stress injury. Am J Sports Med 2007;35:467-74.

53 Brown RA, Mascia A, Kinnear DG, et al. An 18-year review of sports groin injuries in the elite hockey player: clinical presentation, new diagnostic imaging, treatment, and results. Clin J Sport Med 2008;18:221-6.

54 Lloyd DM, Sutton CD, Altafa A, et al. Laparoscopic inguinal ligament tenotomy and mesh reinforcement of the anterior abdominal wall: a new approach for the management of chronic groin pain. Surg Laparosc Endosc Percutan Tech 2008; 18:363-8.

55 Meyers WC, McKechnie A, Philippon MJ, et al. Experience with "sports hernia" spanning two decades. Ann Surg 2008;248:656-64.

56 Radic R, Annear P. Use of pubic symphysis curettage for treatment-resistant osteitis pubis in athletes. Am J Sports Med 2008;36:122-8.

57 Topol GA, Reeves KD. Regenerative injection of elite athletes with career-altering chronic groin pain who fail conservative treatment: a consecutive case series. Am J Phys Med Rehabil 2008;87:890-902.

58 Ziprin P, Prabhudesai SG, Abrahams S, et al. Transabdominal preperitoneal laparoscopic approach for the treatment of sportsman's hernia. J Laparoendosc Adv Surg Tech 2008;18:669-72.

59 Atkinson HD, Johal P, Falworth MS, et al. Adductor tenotomy: its role in the management of sports-related chronic groin pain. Arch Orthop Trauma Surg 2010;130:965-70.

60 Jansen JA, Mens JM, Backx FJ, et al. Changes in abdominal muscle thickness measured by ultrasound are not associated with recovery in athletes with longstanding groin pain associated with resisted hip adduction. J Orthop Sports Phys Ther 2009;39:724-32.

61 Mann $C D$, Sutton CD, Garcea $G$, et al. The inguinal release procedure for groin pain: Initial experience in 73 sportsmen/women. $\mathrm{Br} /$ Sports Med 2009;43:579-83.

62 Schilders E, Talbot JC, Robinson P, et al. Adductor-related groin pain in recreational athletes. Role of the adductor enthesis, magnetic resonance imaging, and entheseal pubic cleft injections. J Bone Joint Surg Am 2009;91:2455-60.

63 Schlegel TF, Bushnell BD, Godfrey J, et al. Success of nonoperative management of adductor longus tendon ruptures in National Football League athletes. Am J Sports Med 2009;37:1394-9.

64 Weir A, Veger SA, Van De Sande HB, et al. A manual therapy technique for chronic adductor-related groin pain in athletes: a case series. Scand J Med Sci Sports 2009;19:616-20

65 Weir A, Jansen J, van Keulen J, et al. Short and mid-term results of a comprehensive treatment program for longstanding adductor-related groin pain in athletes: a case series. Phys Ther Sport 2010;11:99-103.

66 Weir A, Jansen JA, van de Port IG, et al. Manual or exercise therapy for long-standing adductor-related groin pain: a randomised controlled clinical trial. Man Ther 2011;16:148-54.

67 Muschaweck U, Berger L. Minimal Repair technique of sportsmen's groin: an innovative open-suture repair to treat chronic inguinal pain. Hernia 2010;14:27-33. 
68 Chernyavsky VS, Davidov T, Trooskin SZ, et al. Athlete's hernia-a true, early direct inguinal hernia: diagnosis, pathophysiology, and surgical treatment. Am Surg 2011;77:1472-6.

69 Paajanen $H$, Brinck $T$, Hermunen $H$, et al. Laparoscopic surgery for chronic groin pain in athletes is more effective than nonoperative treatment: a randomized clinical trial with magnetic resonance imaging of 60 patients with sportsman's hernia (athletic pubalgia). Surgery (USA) 2011;150:99-107.

70 Preskitt JT. Sports hernia: the experience of Baylor University Medical Center at Dallas. Bayl Univ Med Cent Proc 2011;24:89-91.

71 Robertson IJ, Curran C, McCaffrey N, et al. Adductor tenotomy in the management of groin pain in athletes. Int J Sports Med 2011;32:45-8.

72 Dojcinovic $B$, Sebecic $B$, Staresinic $M$, et al. Surgical treatment of chronic groin pain in athletes. Int Orthop 2012;36:2361-5.

73 Jans C, Messaoudi N, Pauli S, et al. Results of surgical treatment of athletes with sportsman's hernia. Acta Orthop Belg 2012;78:35-40.

74 Maffulli N, Loppini M, Longo UG, et al. Bilateral mini-invasive adductor tenotomy for the management of chronic unilateral adductor longus tendinopathy in athletes. Am J Sports Med 2012;40:1880-6.

75 Messaoudi N, Jans C, Pauli S, et al. Surgical management of sportsman's hernia in professional soccer players. Orthopedics 2012;35:e1371-e5.

76 Comin J, Obaid H, Lammers $\mathrm{G}$, et al. Radiofrequency denervation of the inguinal ligament for the treatment of 'Sportsman's Hernia': a pilot study. Br J Sports Med 2013;47:380-6.

77 Economopoulos KJ, Milewski MD, Hanks JB, et al. Sports hernia treatment: modified bassini versus minimal repair. Sports Health 2013;5:463-9.

78 Garvey JF, Hazard H. Sports hernia or groin disruption injury? Chronic athletic groin pain: a retrospective study of 100 patients with long-term follow-up. Hernia 2013 Published Online First: 12 October 2013.

79 Jakoi A, O'Neill C, Damsgaard C, et al. Sports hernia in National Hockey League players: does surgery affect performance? Am J Sports Med 2013;41:107-10.

80 Mei-Dan 0, Lopez V, Carmont MR, et al. Adductor tenotomy as a treatment for groin pain in professional soccer players. Orthopedics 2013;36:e1189-e97.
81 Schilders E, Dimitrakopoulou A, Cooke M, et al. Effectiveness of a selective partial adductor release for chronic adductor-related groin pain in professional athletes. Am J Sports Med 2013;41:603-7.

82 Bernhardt GA, Gruber G, Molderings BS, et al. Health-related quality of life after TAPP repair for the sportsmen's groin. Surg Endosc 2014;28: 439-46.

83 Cavalli M, Bombini G, Campanelli G. Pubic inguinal pain syndrome: the so-called sports hernia. Surg Technol Int 2014;24:189-94.

84 de Queiroz RD, de Carvalho RT, Szeles PR, et al. Return to sport after surgical treatment for pubalgia among professional soccer players. Rev Brs Ortop 2014;49:233-9.

85 Sansone $\mathrm{M}$, Ahlden $\mathrm{M}$, Jonasson $\mathrm{P}$, et al. Can hip impingement be mistaken for tendon pain in the groin? A long-term follow-up of tenotomy for groin pain in athletes. Knee Surg Sports Traumatol Arthrosc 2014;22:786-92.

86 Crowther M, Lim W, Crowther MA. Systematic review and meta-analysis methodology. Blood 2010;116:3140-6.

87 Tallon C, Coleman BD, Khan KM, et al. Outcome of surgery for chronic Achilles tendinopathy. A critical review. Am J Sports Med 2001;29:315-20.

88 de Vos RJ, van Veldhoven PL, Moen MH, et al. Autologous growth factor injections in chronic tendinopathy: a systematic review. Br Med Bull 2010:95:63-77.

89 Schulz KF, Altman DG, Moher D, et al. CONSORT 2010 statement: updated guidelines for reporting parallel group randomized trials. Ann Intern Med 2010;152:726-32.

90 von Elm E, Altman DG, Egger M, et al. The Strengthening the Reporting of Observational Studies in Epidemiology (STROBE) statement: guidelines for reporting observational studies. Lancet 2007;370:1453-7.

91 Weir A. From disruption to consensus: the thousand mile journey. Br J Sports Med 2014;48:1075-7.

92 Sheen AJ, Stephenson BM, Lloyd DM, et al. 'Treatment of the Sportsman's groin': British Hernia Society's 2014 position statement based on the Manchester Consensus Conference. Br J Sports Med 2014:48:1079-87. 